\title{
In vitro und in vivo Biokompatibilität einer neuartigen, 3-dimensionalen Cellulose-Matrixstruktur
}

\section{In Vitro and In Vivo Biocompatibility of a Novel, 3-Dimensional Cellulose Matrix Structure}

Autoren

Institute

\author{
S. E. Dunda ${ }^{1,2}$, M. Ranker ${ }^{1}$, N. Pallua ${ }^{1}$, H.-G. Machens ${ }^{3}$, A. Ravichandran ${ }^{4}$, J-T. Schantz ${ }^{3}$
}

${ }^{1}$ Plastische Chirurgie, Hand- und Verbrennungschirurgie, Uniklinik RWTH Aachen, Aachen

${ }^{2}$ Plastische Chirurgie und Handchirurgie, St. Marien-Krankenhaus, Berlin

${ }^{3}$ Plastische Chirurgie und Handchirurgie, Klinikum rechts der Isar, München

${ }^{4}$ School of Chemical and Biomedical Engineering, Nanyang Technological University, Singapore

\section{Schlüsselwörter \\ - Neokapillarisierung \\ - Experimentelle Chirurgie \\ - Matrix}

\section{Key words}

- neocapillarisation

- experimental surgery

0 matrix eingereicht 29.3.2015

akzeptiert $\quad 8.6 .2015$

Bibliografie

DOI http://dx.doi.org/

10.1055/s-0035-1555894

Online-Publikation: 29.10.2015

Handchir Mikrochir Plast Chir

2015; 47: 378-383

(c) Georg Thieme Verlag KG

Stuttgart · New York

ISSN 0722-1819

\section{Korrespondenzadresse}

\section{Dr. Sebastian Erol Dunda}

Plastische Chirurgie, Hand- und Verbrennungschirurgie Uniklinik RWTH Aachen Pauwelsstraße 30

52074 Aachen

sdunda@icloud.com

\section{Zusammenfassung}

\section{$\nabla$}

Hintergrund: Die biochemischen und physikalischen Eigenschaften von Matrices sind von entscheidender Bedeutung bei der Geweberegeneration. In dieser Studie wurde die neuartige, aus Cellulose bestehende, 3-dimensionale Matrix Xellulin ${ }^{\circledR}$ angewendet und in Hinblick auf die Biokompatibilität und Eignung zur Geweberegeneration in vitro und in vivo evaluiert.

Material und Methoden: Die zu evaluierende Matrix Xellulin ${ }^{\circledR}$ ist eine natürliche hydrologische Gel-Matrix, die aus bakterieller Cellulose und Wasser besteht. In vitro erfolgte eine Besiedelung und Kultivierung der Matrix mit humanen Fibroblasten zur Evaluation der Biokompatibilität und des Zellverhaltens im Hinblick auf Adhärenz und Proliferation. In vivo wurden Präadipozyten, isoliert aus den inguinalen Fettkörpern von 4 bis 6 Wochen alten Wistar-Ratten, in 3 Cellulose-Matrices unter 3 unterschiedlichen Bedingungen transplantiert: a) mit 500000 Präadipozyten subkutan, b) mit 500000 Präadipozyten vaskularisiert in einem In-vivo-Bioreaktor, c) ohne Präadipozyten subkutan als Kontrolle. Nach Explantation am 14. postoperativen Tag erfolgte die histologische (Zellmorphologie) und immunhistochemische Evaluation (Kapillarisierung).

Ergebnisse: In vitro zeigt sich eine ausgezeichnete Biokompatibilität mit guter Adhärenz der Fibroblasten auf der Matrix mit nachgewiesener Proliferation und Ausbildung eines 3-dimensionalen Zellnetzwerkes. In vivo kann in allen Versuchsgruppen eine Kapillarisierung entlang der vorgegebenen Kapillarbettstrukturen mit Nachweis von Erythrozyten (H/E-Färbung) sowie die Präsenz von Endothelzellen (RECA-1-Immunhistochemie) nachgewiesen werden. Eine signifikant höhere Kapillarisierung zeigt sich in der vaskularisierten Bioreaktor-Gruppe (18,4 vessels/ $100000 \mu^{2}$ (Gruppe b) vs. 8,1 (Gruppe a), $\mathrm{p}<0,05)$. Die Zelldichte ist ebenso in der vaskula-

\section{Abstract \\ $\nabla$}

Background: Biological and physical characteristics of matrices are one essential factor in creating bioartificial tissue. In this study, a new 3-dimensional cellulose matrix (Xellulin ${ }^{\circledR}$ ) was tested in terms of biocompatibility and applicability for tissue engineering in vitro and in vivo. Materials and Methods: The tested matrix Xellulin ${ }^{\circledR}$ is a natural hydrological gel-matrix containing bacterial cellulose and water. To evaluate the cell biocompatibilty, cell adherence and proliferation characteristics in vitro, the matrix was cultured with human fibroblasts. Further in vivo studies were carried out by transplanting preadipocytes of 4- to 6-weekold Wistar rats with 3 different conditions: a) Xellulin ${ }^{\circledR}$ including 500000 preadipocytes subcutaneous, b) Xellulin ${ }^{\circledR}$ including 500000 preadipocytes within an in vivo bioreactor chamber, c) Xellulin ${ }^{\circledR}$ without cells subcutaneous as control. After explantation on day 14 histomorphological and immunohistochemical evaluations were performed.

Results: In vitro study revealed an excellent biocompatibility with good cell adherence of the fibroblasts on the matrix and evidence of cell proliferation and creation of a 3-dimensional cell network. In vivo neocapillarisation could be shown in all groups with evidence of erythrocytes (H/E staining) and endothelial vascular cells (RECA-1-staining). A significantly higher vascular density was shown in vascularised bioreactor group (18.4 vessels/100000 $\mu^{2}$ (group b) vs. 8.1 (group a), p <0.05). Cell density was the highest in the vascularised group, but without significant values. No immunogenic reaction to the matrix was noticed.

Discussion: The promising in vitro results concerning cell adherence and proliferation on the tested matrix could be confirmed in vivo with an evidence of 3-dimensional neocapillarisation. 
risierten Bioreaktor-Gruppe am höchsten, ohne eine Signifikanz zu erzielen. Eine immunogene Reaktion auf die Cellulose-Matrix zeigt sich nicht.

Schlussfolgerung: Die positiven Ergebnisse in vitro bezüglich der Zelladhärenz und Proliferation in der Cellulose-Matrix bestätigt sich in vivo mit zusätzlicher Ausbildung von 3-dimensionalen Kapillarbettstrukturen. Das Zell-Überleben ist nach Tag 14 bei Verwendung des vaskularisierten In-vivo-Bioreaktors verbessert, jedoch nicht signifikant. Eine neuerliche Versuchsserie über einen längeren Zeitraum (28-42 Tage) ist erforderlich, um das langfristige Zell-Überleben und die Stabilitätsvorteile der Cellulose-Matrix als vielversprechende Matrix in der Geweberegeneration zu evaluieren. Des Weiteren sind Studien hinsichtlich des Gewebeeinbaues in umliegende Nachbarstrukturen sowie Langzeit-Biokompatibilitätsstudien notwendig.

\section{Einleitung}

Der Ersatz von funktionell eingeschränktem oder destruiertem Gewebe durch die Transplantation von Zellen oder von artifiziell hergestellten Gewebestrukturen ist nicht zuletzt in der rekonstruktiven Chirurgie von klinischer Bedeutung. Die Herstellung von künstlichem Gewebe erfordert die Transplantation von Vorläuferzellen mit der Fähigkeit, sich zu vollwertigen Zellen innerhalb einer biologischen oder artifiziellen Umgebung zu entwickeln [1]. Ebenso entscheidend für die Entstehung einer 3-dimensionalen Gewebestruktur sind neben der geeigneten Zellwahl jedoch auch andere Faktoren wie die Kapillarisierung und die biomechanischen Eigenschaften der Matrix und die Integration in das umliegende Gewebe. Die Vaskularisierung und somit die Nutrition der transplantierten Zellen erfordert in einem 3-dimensionlane Gewebeblock das Vorhandensein eines Gefäßnetzes. Hierzu sind verschiedene In-vivo-Modelle beschrieben, allem voran das AV-loop Modell oder die axiale Versorgung [2-8].

Die eigentliche Matrix muss neben der Biokompatibilität multiple weitere Eigenschaften vorweisen, um zu gewährleisten, dass die transplantierten Zellen adhärieren, migrieren, proliferieren und sich weiter differenzieren können $[9,10]$. Ein weiteres wesentliches Kriterium ist dabei auch der Vorgang einer gegebenenfallls möglichen Biodegrabilität. Die Matrix sollte dabei ohne Bildung von toxischen Substanzen abbaubar sein, um so ein Einwachsen des sich neu formierenden artifiziellen Gewebes zu ermöglichen [11,12]. Die Matrix sollte auch so beschaffen sein, dass sie Gewebedruck ausreichend standhalten kann, gleichzeitig jedoch über eine ausreichende Porosität verfügt, um die Ausbildung eines Kapillarnetzes zu erlauben $[12,13]$. Dabei ist die Interkonnektivität der Porenstrukturen von entscheidender Bedeutung, sodass ein homogenes Zellwachstum und eine Bereitstellung mit Nährstoffen sowie ein Abtransport von Metaboliten gewährleistet werden kann. Verschiedene Matrices sind bereits umfangreich in einer Vielzahl von Studien eingesetzt und sowohl in vitro als auch in vivo untersucht worden. Hydrogele wie Fibrin, als eines der meist eingesetzten Matrices, haben sich bei entsprechender Konzentration als positiv in Bezug auf das Überleben der transplantierten Zellen erwiesen. Aufgrund der schnellen Degeneration des Fibrin ist es jedoch zur Herstellung einer langfristig stabilen Matrix als alleinige Komponente nicht ausreichend [7,11]. 3-dimensionale Scaffolds wie poly(L-Laktatsäure) (PLLA) zeigen eine deutlich längere Degenerationszeit von bis zu mehreren Monaten und haben als Bestandteil in meh-
Cell survival was higher in the vascularised group, but without significance. Long-term tests (28-42 days) need to be carried out to evaluate long-term cell survival and the matrix stability. Furthermore, studies concerning the implementation of the matrix within anatomic structures as well as long-term biocompatibility are needed.

reren medizinischen Produkten wie Nahtmaterial oder Implantaten bereits Einzug in der medizinischen Anwendung gefunden $[12,14,15]$.

Das in dieser Studie untersuchte Biomaterial Xellulin ${ }^{\circledR}$ besteht aus kristalliner Cellulose, die von Gluconacetobacter xylinus produziert wird, und circa 99\% aus Wasser besteht [16]. Die regelmäßige Abfolge von Cellulosefaserbündeln im Nanometerbereich bildet eine gerüstartige 3-dimensionale Umgebung, die eine ausreichende Diffusion zur Zellnutrition gewährleistet. Cellulose ist als biologisches Produkt im Säugerorganismus inert und in seiner Herstellung sehr flexibel. So konnten im Bereich des Knorpel-Tissue-Engineering vielversprechende Ergebnisse mit einer guten Migration und Besiedlung der Cellulose-Matrix mit Chondrozyten erzielt werden [9]. Bäckdahl et al. beschrieben zuvor bereits eine gute Biokompatibilität der Cellulose, unter anderem in Bezug auf glatte Muskelzellen in der Herstellung von artifiziellen Gefäßprothesen [17,18]. Mit dem Ziel, artifizielles Gewebe für die Anwendung in der Rekonstruktion von Weichteilgewebedefekten herzustellen, wurde in dieser Studie die Biokompatibilität einer 3-dimensionalen, mit Kanälchen strukturierten Cellulose-Matrix in vitro und in vivo evaluiert.

\section{Material und Methoden \\ $\nabla$} Cellulose-Matrix Xellulin ${ }^{\circledR}$

Essigsäurebakterien produzieren unter anderem Exopolysaccharide wie Acetan und Cellulose. Gluconacetobacter xylinus ist dabei der bekannteste Produzent von reiner Cellulose in großen Mengen, wobei die produzierten Cellulosefasern mit einem Fibrillen-Durchmesser von etwa $100 \mathrm{~nm} 100$-mal dünner sind als pflanzliche Cellulosefasern [19]. Trotz dieses geringen Durchmessers verfügen die so synthetisierten Fasern über eine sehr hohe Festigkeit von 0,33 MPa im Zugversuch, was jedoch gleichzeitig mit einer geringen Elastizität einhergeht [20]. Ein Vorteil für den medizinischen Einsatz ist die hohe Temperaturstabilität, da eine thermische Zersetzung erst bei circa $300^{\circ} \mathrm{C}$ eintritt und somit eine Dampfsterilisation möglich ist [21]. Das hier benutzte Verfahren zur Herstellung der Cellulose nutzt eine Mikrofiltrationsmembran zur Trennung des Produzenten Gluconacteobacter xylinus vom Kulturmedium. Das dabei entstehende CellulosePellikel besteht aus mehreren, parallel angeordneten Schichten mit vertikalen Kapillaren im Abstand von $200 \mu \mathrm{m}$ (• Abb. 1) [22]. Die so hergestellte Cellulose-Matrix wird dampfsterilisiert und in PBS (5\%ig) gelagert. 


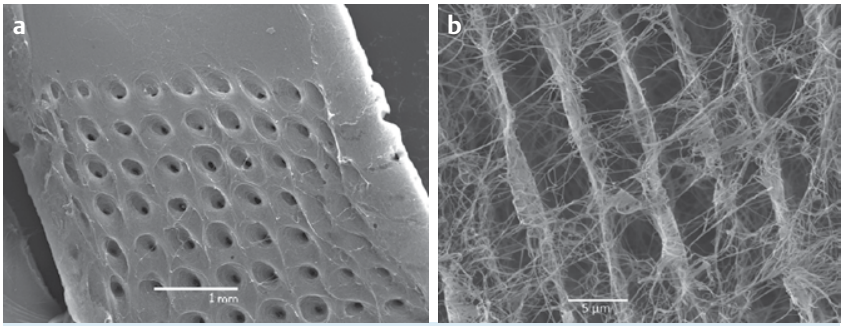

Abb. 1 Elektronenmikroskopische Oberflächen-Darstellung der Cellulose-Matrix mit Nachweis der vertikalen Versorgungskanälchen in einem Abstand von $200 \mu \mathrm{m}$ (a); Querschnitt der Matrix zur Darstellung der Porosität und zusätzlichen horizontal verlaufenden Kanälchen (b).

\section{Zellkultur (Fibroblasten, Adipozyten)}

Humane dermale Fibroblasten wurden aus einer Hautbiopsie isoliert. Die Zellen wurden in Dulbecco's modifiziertem Eagle‘s Medium (Sigma-Aldrich, Irvine, Großbritannien) mit 1\%igem Penicillin-Streptomycin-Gemisch sowie 10\%igem Rinderserum (Viralex, PAA Laboratories, Linz, Österreich) suspendiert. Das Kulturmedium wurde jeden 2.-3. Tag gewechselt und die Zellen in 3 Passagen expandiert, entsprechend gelöst mittels Trypsin $0,25 \%$ und in $\mathrm{T} 75$ Kulturflaschen im Verhältnis 1:3 resuspendiert. Die für die In-vivo-Transplantation verwendeten Adipozyten wurden aus den inguinalen Fettdepots von 4 bis 6 Wochen alten Wistar-Ratten gewonnen. Die explantierten Fettpolster wurden unter sterilen Bedingungen zerkleinert und in PBS gewaschen. Das so verarbeitete Fettgewebe wurde hierauf in einer 3,5\%igen Albumin-Phosphatpuffer-Lösung mit D-Glukose und Kollagenase I für 3 Stunden bei $37^{\circ} \mathrm{C}$ in einem Schüttelwasserbad inkubiert. Nach Zentrifugation des Digestates wurde der mehrschichtige Überstand über dem Zellsediment verworfen. Das gewonnenen Zellsediment wurde erneut zentrifugiert, in PBS resuspendiert und mittels eines Zellsiebes (Falcon Cell Strainer, 70 $\mu \mathrm{m}$ Porengröße) filtriert, die Zellzahl bestimmt und die Präadipozyten in Kulturflaschen ausgesät. Nach Expansion in bis zu 3 Passagen und einer ausreichenden Zellzahl erfolgte die unten beschriebene Transplantation der Zellen. Der histologische Nachweis der Präadipozyten erfolgte mittels Oil-Red Färbung.

\section{In-vitro-Besiedlung der Cellulose-Matrix}

Zur Überprüfung der Biokompatibilität der Cellulose-Matrix wurden diese in der benötigten Anzahl bei $121^{\circ} \mathrm{C}$ für $20 \mathrm{~min}$ sterilisiert und in einer 48-Well-Platte jeweils mit 200000 Fibroblasten und $0,5 \mathrm{ml}$ Medium inkubiert. Die Verlaufskontrolle zur Beurteilung der Zelladhäsion und -migration erfolgte mittels Lichtmikroskopie (Olympus Corporation, Tokyo, Japan) alle 24 Stunden über einen Zeitraum von 15 Tagen. Zur immunhistochemischen Auswertung erfolgte an Tag 1, 3 und 7 jeweils ein BCA-Protein-Assay. Hierzu wurden die besiedelten CelluloseMatrices mit PBS gewaschen und mit $1 \mathrm{ml}$ Kollagenase-Trypsin-Lösung in einem Röhrchen bei $-30^{\circ} \mathrm{C}$ gelagert. Der Nachweis erfolgte hierauf bei einer Wellenlänge von $595 \mathrm{~nm}$ sowohl an den Proben als auch einer entsprechenden Leer-Kontrolle.

Darüber hinaus erfolgte an Tag 1, 4, 8 und 15 ein Alamar-blueAssay zum Nachweis der Zellviabilität. Hierzu wurden die CelluloseMatrices mit 10\%iger Alamar-blue-Lösung für 1 Stunde inkubiert. Der Effloreszenz-Nachweis erfolgte hierauf entsprechend bei $570 \mathrm{~nm}$. Zur strukturellen Analyse erfolgten ergänzend an Tag 7 und 15 die Durchführung elektronenmikroskopischer Untersuchungen (SEM) nach Fixierung der Präparate in 4\%igen Paraformaldehyd (PFA) für 30 min und anschließender Dehydrie- rung in steigender Äthanol-Konzentration (für jeweils 5 min in $50,70,80,90$ und $100 \%$ EtOH).

\section{In-vivo-Transplantation und Versuchsgruppen}

4-6 Wochen alte, männliche Wistar-Ratten wurden entsprechend des genehmigten Tierversuchsprotokolls im Institut für Tierversuchskunde an der Uniklinik RWTH Aachen bei $21^{\circ} \mathrm{C}$ unter Tag-Nacht-Rhythmus gehalten und versorgt. An jedem der Tiere erfolgte die Implantation von 3 Cellulose-Matrices in folgender Einteilung: a) mit 500000 Präadipozyten subkutan, b) mit 500000 Präadipozyten vaskularisiert in einem In-vivoBioreaktor unter Verwendung der Vasa epigastrica als axiale Versorgungsgefäße der Cellulose-Matrix, c) ohne Präadipozyten subkutan als Kontrolle. Nach Explantation am 14. postoperativen Tag erfolgte die histologische (Zellmorphologie mittels $\mathrm{H} / \mathrm{E}$ und Oil Red) sowie immunhistochemische Evaluation (Kapillarisierung mittels RECA-1).

\section{Implantation der Cellulose-Matrices}

Die Implantation der Cellulose-Matrices erfolgt wie vorbeschrieben in Wistar-Ratten unter 3 unterschiedlichen Bedingungen. Hierzu wurden die Ratten mittels intraperitonealer Applikation von Ketamin (10\%ig, 0,06 ml/100g KG, Medistar Ascheberg, Deutschland) und Medetomidine $(0,03 \mathrm{ml} / 100 \mathrm{~g} \mathrm{KG}$, Pfizer, Paris, Frankreich) anästhesiert. Nach sterilem Abwaschen der OPGebiete erfolgte die Hautinzision im Sinne einer Laparatomie. Nach Dissektion der Rectus-Muskulatur erfolgte lupenmikroskopische die Darstellung und Freilegung der Vasa epigastrica inferior und superior auf der kompletten Länge. Hierauf erfolgte die Implantation des In-vivo-Bioreaktors mit der Vasa epigastrica als zentrales Versorgungsgefäß wie von Dunda et al. zuvor beschrieben [7]. Nach der Implantation der Cellulose-Matrix in die Bioreaktor-Kammer wurden 500000 Präadipozyten in einem Gesamtvolumen von 0,3 $\mathrm{ml}$ PBS der Cellulose-Matrix hinzugefügt und die Bioreaktor-Kammer vollständig verschlossen. Der Wundverschluss erfolgte mehrschichtig mit Adaptation der RectusMuskulatur (Vicryl 4.0, Ethicon, Norderstedt, Deutschland) sowie Hautnaht (Prolene 4.0, Ethicon, Norderstedt, Deutschland). Zur subkutanen Implantation der Cellulose-Matrices erfolgte die Hautinzision mit Präparation einer epifaszialen Tasche im Bereich beider Flanken. Hiernach erfolgte die Zugabe von erneut 500000 Präadipozyten zu einer der beiden Cellulose-Matrices mit der verbliebenden ohne Zellen als Kontrolle.

\section{Histologie und Immunhistochemie}

Am 14. postoperativen Tag erfolgte die Explantation der Matrices nach erneuter intraperitonealer Anästhesie und anschließend die Aufarbeitung der Explantate mit entsprechender Einbettung für die spätere histologischen und immunhisto-chemischen Untersuchungen. Nach der Explantation wurden die Wistar-Ratten durch eine intrakardiale Kalium-Injektion sakrifiziert. Die H/E-Färbung erfolgte, um die histomorphologische Struktur der Zellen in der Cellulose-Matrix sowie eine mögliche Neokapillarisierung zu beurteilen. Zur Evaluierung des Zellüberlebens der Präadipozyten erfolgte zudem die Durchführung von Oil-Red-Färbungen.

Um eine Neovaskularisierung nachzuweisen und die Kapillaraussprossung quantitativ beurteilen zu können, erfolgte darüber hinaus die immunhistochemische Färbung von Gefäßendothelzellen unter Verwendung von RECA-1 (Abcam Inc., Cambridge, MA, USA). Hierzu wurden Kryoschnitte mit $1 \%$ igem Aceton (Merck, Darmstadt, Deutschland) für $5 \mathrm{~min}$ bei $25^{\circ} \mathrm{C}$ fixiert und 


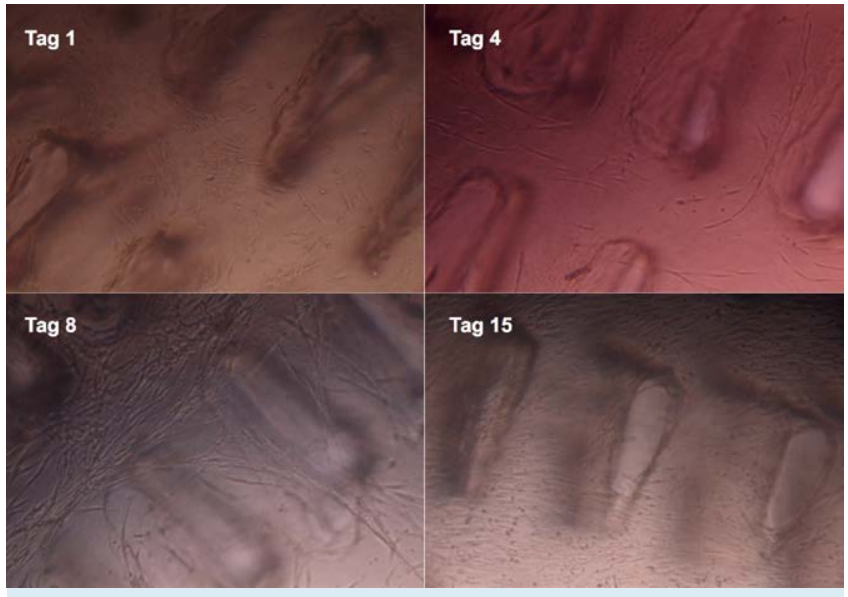

Abb. 2 Lichtmikroskopische Verlaufskontrolle der in vitro besiedelten Cellulose-Matrix mit Darstellung der Zelladhäsion und Proliferation im Zeitverlauf von Tag 1 bis Tag 15.

im Anschluss mit dem RECA-1 Antikörper in einer Verdünnung von 1:25 für $30 \mathrm{~min}$ ebenfalls bei $25^{\circ} \mathrm{C}$ inkubiert. Im Anschluss erfolgte die Applikation eines sekundären Alexa Fluor-488 nmAntikörpers mit erneuter Inkubation von $30 \mathrm{~min}$.

Sämtliche histologischen sowie immunhistochemischen Färbungen wurden untersucht und dokumentiert mittels Lichtund Fluoreszenz-Mikroskopie unter Verwendung eines BZ-9000 Mikroskops (Keyence, Osaka, Japan). Die Auszählung der RECA1-markierten Endothelzellen erfolgte mithilfe des Keyence Analyzing Software (Keyence, Osaka, Japan) und wurde dokumentiert in Kapillare pro $100000 \mu \mathrm{m}^{2}$.

\section{Statistische Analyse}

Die statistische Analyse der quantitativen Auswertung der Neokapillarisierung (immunhistochemische Färbung mit RECA-1) erfolgte mittels $t$-test unter Verwendung von GraphPad Prism 6 (GraphPad Software, La Jolla, CA, USA). Ergebnisse mit einem p-Wert von $p<0,05$ wurden als signifikant eingestuft.

\section{Ergebnisse}

Die In-vitro-Kultivierung der Fibroblasten auf den CelluloseMatrices zeigte in der lichtmikroskopischen Verlaufskontrolle neben einer Adhäsion der Zellen eine im Zeitverlauf von Tag 1 bis Tag 15 zunehmende Proliferation der Fibroblasten sowie die deutliche Ausbildung eines Zellnetzwerkes auf der Matrix (○ Abb. 2). Diese Beobachtungen ließen sich durch die zunehmenden Proteinnachweis mittels des BCA-Assays bestätigen mit einer Absorptionsrate von 0,69 an Tag 1 und von 0,81 an Tag 7 ( $\odot$ Abb. 3a). Gleich verhält es sich mit dem Nachweis der Zellviabilität im Alamar-blue-Assay mit einer ansteigenden Absorptionsrate von 0,043 an Tag 1 auf 0,099 an Tag 15 ( $\bullet$ Abb. 3b) und somit indirekt einer steigenden Zahl an vitalen Zellen.

Die histologische Auswertung der in vivo transplantierten Cellulose-Matrices zeigte in den H/E-Färbungen den Nachweis von vitalen Zellen in den Matrices unabhängig von den Transplantationsgruppen ( $\bullet$ Abb. 4a-c). Auffällig ist eine deutliche zelluläre Infiltration der Cellulose-Matrices im Sinne einer Gewebeintegration in den mit Präadipozyten transplantierten Matrices. Demgegenüber erscheint die Zellbesiedlung der Kontrolle (ohne Präadipozyten) auf die oberflächlichen Schichten der Cellulose-



Abb. 3 Biochemischer Nachweis der Zellproliferation durch zunehmenden Proteinnachweis mittels der BCA-Reaktion mit einer Absorptionsrate von 0,69 an Tag 1 und von 0,81 an Tag 7 a; Nachweis der Zellviabilität im Alamar-blue-Assay mit einer ansteigenden Absorptionsrate von 0,043 an Tag 1 auf 0,099 an Tag 15 b und somit indirekt einer steigenden Zahl an vitalen Zellen.

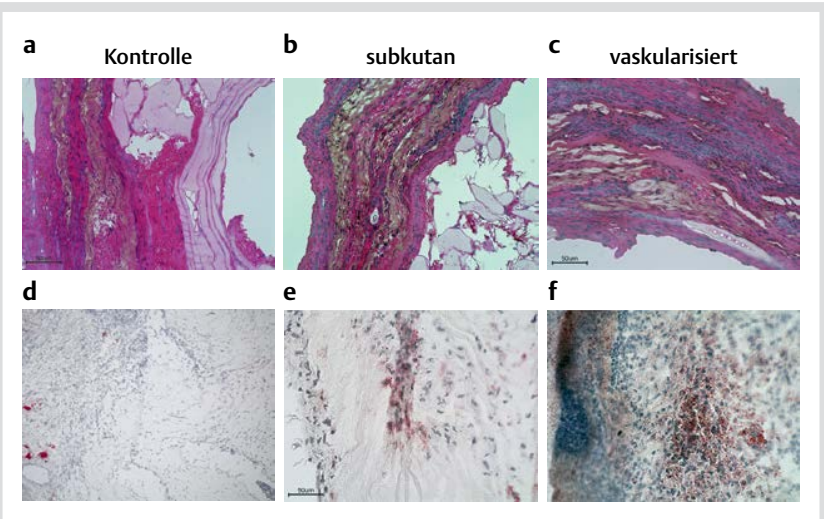

Abb. $4 \mathrm{H} / \mathrm{E}$-Färbungen mit Nachweis von vitalen Zellen in den Matrices unabhängig von den Transplantationsgruppen (a-c); Oil-Red-Färbung mit Nachweis von vitalen Präadipozyten nur in den Matrices der Gruppen a (vaskularisiert, f) und b (subkutan, e), wobei sich in der vaskularisierten Gruppe deutlich mehr Präadipozyten nachweisen lassen.

Matrix beschränkt. Entsprechend zeigte sich auch in der OilRed-Färbung der Nachweis von vitalen Präadipozyten nur in den Matrices der Gruppen a und b, wobei sich in der vaskularisierten Gruppe (Gruppe b) deutlich mehr Präadipozyten nachweisen lassen ( $\bullet$ Abb. 4d-f). 


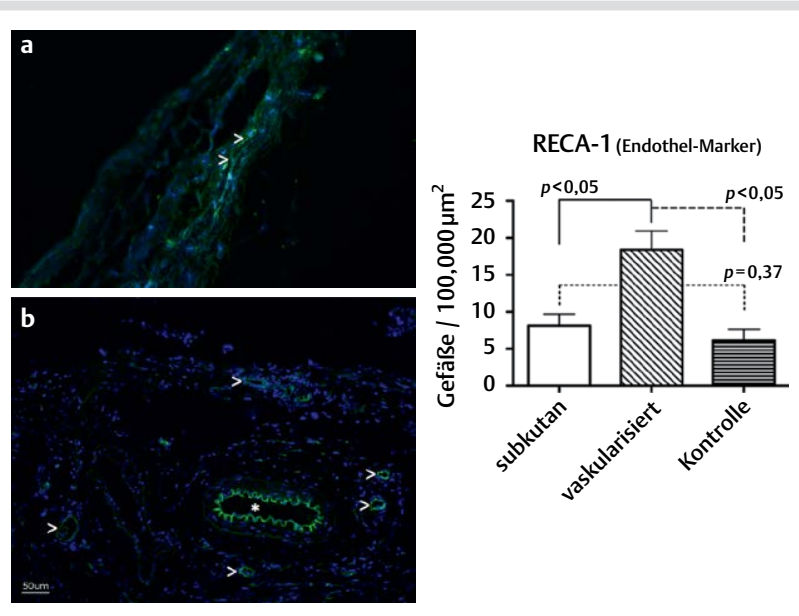

Abb. 5 Immunhistochemische RECA-1-Färbungen mit direktem Nachweis von Gefäßendothelzellen in allen Gruppen (siehe „> “ in a (Gruppe a, subkutan) und b (Gruppe b, vaskularisiert); „ "“ entspricht der Vasa epigastrica als zentrales Bioreaktor-Gefäß); die statistische Auswertung ergibt eine signifikant höheren Endothel-Nachweis in der vaskularisierten Gruppe (b sowie Grafik).

Zusätzlich zeigen sich in den histologischen Färbungen in allen Gruppen Zeichen einer Neovaskularisierung mit multipler Kapillarbildung und Nachweis von Erythrozyten. Dies bestätigte sich in den immunhistochemischen RECA-1-Färbungen mit direktem Nachweis von Gefäßendothelien in allen Gruppen ( $\bullet$ Abb. 5). Die quantitativen Bestimmungen zeigten darüber hinaus eine signifikant höhere Anzahl an Neokapillaren in der vaskularisierten Gruppe im Vergleich zu den subkutan implantierten Cellulose-Matrices (18,4 gegenüber 8,1 Gefäße/100000 $\mu^{2}$, $\mathrm{p}<0,05$, ( $\odot$ Tab. 1). Ein signifikanter Unterschied zwischen den subkutan implantierten Matrices (mit und ohne Präadipozyten) konnte nicht nachgewiesen werden $(p=0,37, \diamond$ Tab. 1).

\section{Diskussion}

In den vergangenen Jahren ist ein wesentlicher Focus im Bereich des Tissue Engineering neben der Zellauswahl und der Vaskularisierung des artifiziellen Gewebes zunehmend auf die Optimierung der Matrix gelegt worden. Die Auswahl der zu transplantierenden Zellen ist grundsätzlich von dem Ziel des zu entwickelnden Gewebes abhängig, wobei sich Präadipozyten als mesenchymale Stammzellen aufgrund ihrer Differenzierbarkeit und Multipotenz als besonders geeignet herausgestellt haben und entsprechend auch in dieser Forschungsarbeit in vivo gewonnen und transplantiert worden sind [23-25].

Wie zuvor beschrieben muss eine für die Zelltransplantation und Geweberegenerierung geeignete Matrix über multiple Eigenschaften verfügen. Hierbei sind neben der erforderlichen Biokompatibilität und Stabilität vor allem die Biodegrabilität sowie eine ausreichend poröse Struktur zur Ermöglichung der Zellmigration und der Neovaskularisierung [9-13].
Tab. 1 Einteilung der in vivo Versuchsgruppen mit Ergebnissen der nachgewiesenen Gefäßendothelzellen (RECA-1-Immunhistochemie).

\begin{tabular}{lc} 
Gruppen in vivo & RECA-1 (Gefäße/100000 $\mathbf{\mu m}^{\mathbf{2}}$ ) \\
$\begin{array}{l}\text { a. subkutan } \\
\text { mit Präadipozyten (500000) } \\
(\mathrm{n}=6)\end{array}$ & $8,13 \pm 1,55$ \\
$\begin{array}{l}\text { b. vaskularisiert } \\
\text { mit Präadipozyten (500000) } \\
(n=6)\end{array}$ & $18,41^{*} \pm 2,51$ \\
\hline $\begin{array}{l}\text { c. subkutan (Kontrolle) } \\
\text { ohne Präadipozyten } \\
(n=6)\end{array}$ & $6,15 \pm 1,47$ \\
$* p<0,05$ gegenüber Gruppe a (subkutan) und c (Kontrolle)
\end{tabular}

Mikrobiologisch hergestellte Cellulose ist als biologisches Matrixmaterial in Bezug auf die Biokompatibilität in mehreren Studien mit positiven Ergebnissen untersucht worden [18-22]. Insbesondere im Bereich des Knorpel-Tissue-Engineering konnten dabei zudem vielversprechende Ergebnisse in puncto Stabilität und Zellmigration erzielt werden $[9,10,15]$. Auch in der hier vorliegenden Arbeit konnten diese Eigenschaften sowohl in vitro als auch in vivo nachgewiesen werden. Die positiven In-vitroErgebnisse bezüglich der Zelladhärenz und Proliferation der verwendeten Fibroblasten in der Cellulose-Matrix sind wesentliche Voraussetzung zur Gewebsintegration einer Matrix. Diese bestätigte sich in vivo mit einer hohen Zellüberlebensrate der transplantierten Adipozyten sowie insbesondere durch die ausgeprägte Neovaskularisierung in allen Versuchsgruppen.

Die Ausbildung von 3-dimensionalen Kapillarbettstrukturen in den nicht axial vaskularisierten Gruppen ist ein ausgesprochen positives Ergebnis bezüglich der 3-dimensionalen Struktur der Cellulose-Matrix, die diese Kapillarisierung ermöglicht. Die darüber hinaus signifikant höhere Neokapillarisierung in der axial vaskularisierten Gruppe bestätigt die Funktionalität und Einsetzbarkeit des von Dunda et al. beschriebenen In-vivo-Bioreaktors $[7,8]$.

Das Zell-Überleben ist nach Tag 14 bei Verwendung des vaskularisierten In-vivo-Bioreaktors zwar verbessert, jedoch nicht signifikant. Eine neuerliche Versuchsserie über einen längeren Zeitraum (28-42 Tage) ist erforderlich, um die langfristige Zell-Überlebensrate und die Stabilitätsvorteile der CelluloseMatrix als vielversprechende Matrix in der Geweberegeneration zu evaluieren. Des Weiteren sind Studien hinsichtlich des Gewebeeinbaues in umliegende Nachbarstrukturen sowie LangzeitBiokompatibilitätsstudien notwendig.

\section{Danksagung}

Die Autoren danken Dr. rer. nat. Günter Berthold und Dr. Friedo Schröder (Xellutec GmbH, Neuried, Deutschland) für die sachkundige und finanzielle Unterstützung. Andrea Fritz und Martina Tappe danken wir für die technische Unterstützung bei der Durchführung der histologischen und immunhistochemischen Untersuchungen. 


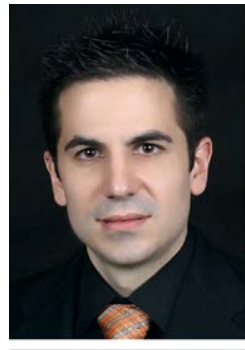

Sebastian E. Dunda

Nach Beendigung seines Studiums an der Medizinischen Hochschule Hannover und der Harvard Medical School in Cambridge 2004, sowie dem erfolgreichen Abschluss der Promotion Facharztweiterbildung zunächst im Fachbereich der Herz-, Thorax- und Gefäßchirurgie in Hannover und Bad Bevensen.

Forschungsaufenthalt an der National University of Singapore (NUS, 2008-2009) mit dem Schwerpunkt des kardialen Tissue Engineering und der Mikrochirurgie. April 2009 Klinik für Plastische Chirurgie, Hand- und Verbrennungschirurgie an die Uniklinik RWTH Aachen. 2014 den Facharzt für Plastische und Ästhetische Chirurgie Forschungsschwerpunkt muskuloskeletale Tissue Engineering mit der Entwicklung eines in vivo Bioreaktor-Modells. Seit Ende 2014 Facharzt in der Klinik für Plastische Chirurgie, Rekonstruktive Mikrochirurgie und Handchirurgie am St. Marien-Krankenhaus in Berlin, zwischenzeitig als kommissarischer Leiter.

\section{Interessenkonflikt: Nein}

\section{Literatur}

1 Mooney DJ, Mikos AG. Growing new organs. Sci Am 1999; 280: 60-65

2 Nomi M, Atala A, Coppi PD et al. Principals of neovascularization for tissue engineering. Mol Aspects Med 2002; 23: 463-483

3 Novosel EC, Kleinhans C, Kluger PJ. Vascularization is the key challenge in tissue engineering. Adv Drug Deliv Rev 2011; 63: 300-311

4 Erol OO, Spira M. New capillary bed formation with a surgically constructed arteriovenous fistula. Plast Reconstr Surg 1980; 66: 109-115

5 Tanaka Y, Sung KC, Tsutsumi A et al. Tissue engineering skin flaps: which vascular carrier, arteriovenous shunt loop or arteriovenous bundle, has more potential for angiogenesis and tissue regeneration? Plast Reconstr Surg 2003; 112: 1636-1644

6 Bach AD, Arkudas A, Tjiawi J et al. A new approach to tissue engineering of vascularized skeletal muscle. J Cell Mol Med 2006; 10: 716-726

7 Dunda SE, Schriever T, Rosen $C$ et al. A new approach of in vivo Musculoskeletal Tissue Engineering using the epigastric artery as central core vessel of a 3-dimensional construct. Plast Surg Int 2012; doi: $10.1155 / 2012 / 510852$
8 Dunda SE, Krings $L K$, Ranker MF et al. Immunocompromising Therapy Increases in vivo Cell Survival in Musculoskeletal Tissue Engineering. J Med Biol Eng 2014; doi:10.5405/jmbe.1924

9 Andersson J, Stenhamre $H$, Bäckdahl $H$ et al. Behavior of human chondrocytes in engineered porous bacterial cellulose scaffolds. J Biomed Mater Res A 2010; 94: 1124-1132

10 Brittberg $M$, Lindahl A, Nilsson A et al. Treatment of deep cartilage defects in the knee with autologous chondrocyte transplantation. N Engl J Med 1994; 331: 889-895

11 Rossi CA, Pozzobon M, De Coppi P. Advances in musculoskeletal tissue engineering: Moving towards therapy. Organogenesis 2010; 6: 167-172

12 Ulery BD, Nair LS, Laurencin CT. Biomedical Applications of Biodegradable Polymers. J Polym Sci B Polym Phys 2011; 49: 832-864

13 Lloyd $A W$. Interfacial bioengineering to enhance surface biocompatibility. Medical Device Technology 2002; 13: 18-21

14 Pillai CKS, Sharma CP. Review Paper: Absorbable Polymeric Surgical Sutures: Chemistry, Production, Properties, Biodegradability, and Performance. J Biomater Appl 2010; 25: 291-366

15 Middleton JC, Tipton AJ. Synthetic biodegradable polymers as orthopedic devices. Biomaterials 2000; 21: 2335-2346

16 Klemm D, Kramer F, Moritz $S$ et al. Nanocelluloses: a new family of nature-based materials. Angew Chem Int Ed Engl 2011; 50: 5438-5466

17 Bäckdahl H, Helenius G, Bodin A et al. Mechanical properties of bacterial cellulose and interactions with smooth muscle cells. Biomaterials 2006; 27: 2141-2149

18 Helenius G, Bäckdahl $H$, Bodin A et al. In vivo biocompatibility of bacterial cellulose. J Biomed Mater Res A 2006; 76: 431-438

19 Ross $P$, Mayer R, Benziman M. Cellulose biosynthesis and function in bacteria. Microbiol Rev 1991; 55: 35-58

20 Bäckdahl H, Esguerra M, Delbro D et al. Engineering microporosity in bacterial cellulose scaffolds. J Tissue Eng Regen Med 2008; 2: 320-330

21 George J, Ramana K, Sabapathy S et al. Characterization of chemically treated bacterial (acetobacter xylinum) biopolymer: Some thermomechanical properties. Int Biol Macromol 2005; 37: 189-194

22 Hofinger M, Bertholdt G, Weuster-Botz D. Microbial production of homogeneously layered cellulose pellicles in a membrane bioreactor. Biotechnol Bioeng 2011; 108: 2237-2240

23 Karagianni $M$, Kraneburg $U$, Klüter $\mathrm{H}$ et al. Autologous fat grafts and supportive enrichment with adipose tissue stromal cells. Handchir Mikrochir Plast Chir 2013; 45: 93-98

24 Wiggenhauser PS, Müller DF, Melchels FP et al. Engineering of vascularized adipose constructs. Cell Tissue Res 2012; 347: 747-757

25 Tsuji W, Rubin JP, Marra KG. Adipose-derived stem cells: Implications in tissue regeneration. World J Stem Cells 2014; 6: 312-321 